\title{
On a new subclass of bi-univalent functions satisfying subordinate conditions
}

Şahsene Altınkaya

Department of Mathematics,

Faculty of Arts and Science, Uludag University, Bursa, Turkey. email: sahsene@uludag.edu.tr
Sibel Yalçın

Department of Mathematics, Faculty of Arts and Science, Uludag University, Bursa, Turkey. email: syalcin@uludag.edu.tr

\begin{abstract}
In the present investigation, we find estimates on the coefficients $\left|a_{2}\right|$ and $\left|a_{3}\right|$ for functions in the function class $S_{\Sigma}(\lambda, \phi)$. The results presented in this paper improve or generalize the recent work of Magesh and Yamini [15].
\end{abstract}

\section{Introduction and definitions}

Let $A$ denote the class of analytic functions in the unit disk

$$
\mathrm{U}=\{z \in \mathbb{C}:|z|<1\}
$$

that have the form

$$
f(z)=z+\sum_{n=2}^{\infty} a_{n} z^{n} .
$$

Further, by $S$ we shall denote the class of all functions in $A$ which are univalent in $\mathrm{U}$.

The Koebe one-quarter theorem [8] states that the image of $U$ under every function $f$ from $S$ contains a disk of radius $\frac{1}{4}$. Thus every such univalent function has an inverse $f^{-1}$ which satisfies

$$
f^{-1}(f(z))=z, \quad(z \in U)
$$

2010 Mathematics Subject Classification: 30C45, 30C50

Key words and phrases: analytic and univalent functions, bi-univalent functions, bistarlike and bi-convex functions, coefficient bounds, subordination 
and

$$
f\left(f^{-1}(w)\right)=w, \quad\left(|w|<r_{0}(f), r_{0}(f) \geq \frac{1}{4}\right)
$$

where

$$
f^{-1}(w)=w-a_{2} w^{2}+\left(2 a_{2}^{2}-a_{3}\right) w^{3}-\left(5 a_{2}^{3}-5 a_{2} a_{3}+a_{4}\right) w^{4}+\cdots .
$$

A function $f(z) \in A$ is said to be bi-univalent in $U$ if both $f(z)$ and $f^{-1}(z)$ are univalent in $\mathrm{U}$.

If the functions $f$ and $g$ are analytic in $\mathrm{U}$, then $f$ is said to be subordinate to $g$, written as

$$
\mathrm{f}(z) \prec \mathrm{g}(z), \quad(z \in \mathrm{U})
$$

if there exists a Schwarz function $w(z)$, analytic in $\mathrm{U}$, with

$$
w(0)=0 \text { and } \quad|w(z)|<1 \quad(z \in \mathrm{U})
$$

such that

$$
f(z)=g(w(z)) \quad(z \in \mathcal{U}) .
$$

Let $\Sigma$ denote the class of bi-univalent functions defined in the unit disk $\mathrm{U}$. For a brief history and interesting examples in the class $\Sigma$, (see [20]).

Lewin [14] studied the class of bi-univalent functions, obtaining the bound 1.51 for modulus of the second coefficient $\left|a_{2}\right|$. Subsequently, Brannan and Clunie [5] conjectured that $\left|a_{2}\right| \leq \sqrt{2}$ for $f \in \Sigma$. Netanyahu [16] showed that $\max \left|a_{2}\right|=\frac{4}{3}$ if $f(z) \in \Sigma$.

Brannan and Taha [4] introduced certain subclasses of the bi-univalent function class $\Sigma$ similar to the familiar subclasses. $S^{\star}(\alpha)$ and $K(\alpha)$ of starlike and convex function of order $\alpha(0<\alpha \leq 1)$ respectively (see [16]). Thus, following Brannan and Taha [4], a function $f(z) \in A$ is the class $S_{\Sigma}^{\star}(\alpha)$ of strongly bi-starlike functions of order $\alpha(0<\alpha \leq 1)$ if each of the following conditions is satisfied:

$$
f \in \Sigma, \quad\left|\arg \left(\frac{z f^{\prime}(z)}{f(z)}\right)\right|<\frac{\alpha \pi}{2} \quad(0<\alpha \leq 1, z \in \mathrm{U})
$$

and

$$
\left|\arg \left(\frac{w g^{\prime}(w)}{g(w)}\right)\right|<\frac{\alpha \pi}{2} \quad(0<\alpha \leq 1, w \in U)
$$


where $g$ is the extension of $f^{-1}$ to $U$. The classes $S_{\Sigma}^{\star}(\alpha)$ and $K_{\Sigma}(\alpha)$ of bi-starlike functions of order $\alpha$ and bi-convex functions of order $\alpha$, corresponding to the function classes $S^{\star}(\alpha)$ and $K(\alpha)$, were also introduced analogously. For each of the function classes $S_{\Sigma}^{\star}(\alpha)$ and $K_{\Sigma}(\alpha)$, they found non-sharp estimates on the initial coefficients. Recently, many authors investigated bounds for various subclasses of bi-univalent functions ([1], [3], [7], [9], [13], [15], [20], [21], [22]).

Not much is known about the bounds on the general coefficient $\left|a_{n}\right|$ for $n \geq$ 4. In the literature, the only a few works determining the general coefficient bounds $\left|a_{n}\right|$ for the analytic bi-univalent functions ([2], [6], [10], [11], [12]). The coefficient estimate problem for each of $\left|a_{n}\right|(n \in \mathbb{N} \backslash\{1,2\} ; \quad \mathbb{N}=\{1,2,3, \ldots\})$ is still an open problem.

In this paper, by using the method [17] different from that used by other authors, we obtain bounds for the coefficients $\left|a_{2}\right|$ and $\left|a_{3}\right|$ for the subclasses of bi-univalent functions considered Magesh and Yamini and get more accurate estimates than that given in [15].

\section{Coefficient estimates}

In the following, let $\phi$ be an analytic function with positive real part in $\mathrm{U}$, with $\phi(0)=1$ and $\phi^{\prime}(0)>0$. Also, let $\phi(\mathrm{U})$ be starlike with respect to 1 and symmetric with respect to the real axis. Thus, $\phi$ has the Taylor series expansion

$$
\phi(z)=1+\mathrm{B}_{1} z+\mathrm{B}_{2} z^{2}+\mathrm{B}_{3} z^{3}+\cdots \quad\left(\mathrm{B}_{1}>0\right) .
$$

Suppose that $u(z)$ and $v(w)$ are analytic in the unit disk $\mathrm{U}$ with $u(0)=$ $v(0)=0,|u(z)|<1,|v(w)|<1$, and suppose that

$$
u(z)=b_{1} z+\sum_{n=2}^{\infty} b_{n} z^{n}, \quad v(w)=c_{1} w+\sum_{n=2}^{\infty} c_{n} w^{n} \quad(|z|<1) .
$$

It is well known that

$$
\left|b_{1}\right| \leq 1, \quad\left|b_{2}\right| \leq 1-\left|b_{1}\right|^{2}, \quad\left|c_{1}\right| \leq 1, \quad\left|c_{2}\right| \leq 1-\left|c_{1}\right|^{2} .
$$

Next, the equations (2) and (3) lead to

$$
\phi(u(z))=1+B_{1} b_{1} z+\left(B_{1} b_{2}+B_{2} b_{1}^{2}\right) z^{2}+\cdots, \quad|z|<1
$$

and

$$
\phi(v(w))=1+\mathrm{B}_{1} \mathrm{c}_{1} w+\left(\mathrm{B}_{1} \mathrm{c}_{2}+\mathrm{B}_{2} \mathrm{c}_{1}^{2}\right) w^{2}+\cdots, \quad|w|<1 .
$$


Definition 1 A function $\mathrm{f} \in \Sigma$ is said to be $\mathrm{S}_{\Sigma}(\lambda, \phi), 0 \leq \lambda \leq 1$, if the following subordination hold

$$
\frac{z f^{\prime}(z)+\left(2 \lambda^{2}-\lambda\right) z^{2} f^{\prime \prime}(z)}{4\left(\lambda-\lambda^{2}\right) z+\left(2 \lambda^{2}-\lambda\right) z f^{\prime}(z)+\left(2 \lambda^{2}-3 \lambda+1\right) f(z)} \prec \phi(z)
$$

and

$$
\frac{w g^{\prime}(w)+\left(2 \lambda^{2}-\lambda\right) w^{2} g^{\prime \prime}(w)}{4\left(\lambda-\lambda^{2}\right) w+\left(2 \lambda^{2}-\lambda\right) w g^{\prime}(w)+\left(2 \lambda^{2}-3 \lambda+1\right) g(w)} \prec \phi(w)
$$

where $\mathrm{g}(w)=\mathrm{f}^{-1}(w)$.

Theorem 1 Let $\mathrm{f}$ given by (1) be in the class $\mathrm{S}_{\Sigma}(\lambda, \phi)$. Then

$$
\left|a_{2}\right| \leq \frac{B_{1} \sqrt{B_{1}}}{\sqrt{\left|\left(12 \lambda^{4}-28 \lambda^{3}+15 \lambda^{2}+2 \lambda+1\right) B_{1}^{2}-\left(1+3 \lambda-2 \lambda^{2}\right)^{2} B_{2}\right|+\left(1+3 \lambda-2 \lambda^{2}\right)^{2} B_{1}}}
$$

and

$$
\left|\mathrm{a}_{3}\right| \leq\left\{\begin{array}{cc}
\frac{\mathrm{B}_{1}}{2\left(2 \lambda^{2}+1\right)} ; & \text { if } \mathrm{B}_{1} \leq \frac{\left(1+3 \lambda-2 \lambda^{2}\right)^{2}}{2\left(2 \lambda^{2}+1\right)} \\
\frac{\left|\left(12 \lambda^{4}-28 \lambda^{3}+15 \lambda^{2}+2 \lambda+1\right) B_{1}^{2}-\left(1+3 \lambda-2 \lambda^{2}\right)^{2} \mathrm{~B}_{2}\right| \mathrm{B}_{1}+2\left(2 \lambda^{2}+1\right) \mathrm{B}_{1}^{3}}{2\left(2 \lambda^{2}+1\right)\left[\left|\left(12 \lambda^{4}-28 \lambda^{3}+15 \lambda^{2}+2 \lambda+1\right) \mathrm{B}_{1}^{2}-\left(1+3 \lambda-2 \lambda^{2}\right)^{2} \mathrm{~B}_{2}\right|+\left(1+3 \lambda-2 \lambda^{2}\right)^{2} \mathrm{~B}_{1}\right]} & \text { if } \mathrm{B}_{1}>\frac{\left(1+3 \lambda-2 \lambda^{2}\right)^{2}}{2\left(2 \lambda^{2}+1\right)} .
\end{array}\right.
$$

Proof. Let $f \in S_{\Sigma}(\lambda, \phi), 0 \leq \lambda \leq 1$. Then there are analytic functions $u, v: U \rightarrow U$ given by (3) such that

$$
\frac{z f^{\prime}(z)+\left(2 \lambda^{2}-\lambda\right) z^{2} f^{\prime \prime}(z)}{4\left(\lambda-\lambda^{2}\right) z+\left(2 \lambda^{2}-\lambda\right) z f^{\prime}(z)+\left(2 \lambda^{2}-3 \lambda+1\right) f(z)}=\phi(u(z))
$$

and

$$
\frac{w g^{\prime}(w)+\left(2 \lambda^{2}-\lambda\right) w^{2} g^{\prime \prime}(w)}{4\left(\lambda-\lambda^{2}\right) w+\left(2 \lambda^{2}-\lambda\right) w g^{\prime}(w)+\left(2 \lambda^{2}-3 \lambda+1\right) g(w)}=\phi(v(w))
$$

where $g(w)=f^{-1}(w)$. Since

$$
\begin{aligned}
& \frac{z f^{\prime}(z)+\left(2 \lambda^{2}-\lambda\right) z^{2} f^{\prime \prime}(z)}{4\left(\lambda-\lambda^{2}\right) z+\left(2 \lambda^{2}-\lambda\right) z f^{\prime}(z)+\left(2 \lambda^{2}-3 \lambda+1\right) f(z)} \\
= & 1+\left(1+3 \lambda-2 \lambda^{2}\right) a_{2} z \\
& +\left[\left(12 \lambda^{4}-28 \lambda^{3}+11 \lambda^{2}+2 \lambda-1\right) a_{2}^{2}+\left(4 \lambda^{2}+2\right) a_{3}\right] z^{2}+\cdots
\end{aligned}
$$


and

$$
\begin{aligned}
& \frac{w g^{\prime}(w)+\left(2 \lambda^{2}-\lambda\right) w^{2} g^{\prime \prime}(w)}{4\left(\lambda-\lambda^{2}\right) w+\left(2 \lambda^{2}-\lambda\right) w g^{\prime}(w)+\left(2 \lambda^{2}-3 \lambda+1\right) g(w)} \\
= & 1-\left(1+3 \lambda-2 \lambda^{2}\right) a_{2} w \\
& +\left[\left(12 \lambda^{4}-28 \lambda^{3}+19 \lambda^{2}+2 \lambda+3\right) a_{2}^{2}-\left(4 \lambda^{2}+2\right) a_{3}\right] w^{2}+\cdots,
\end{aligned}
$$

it follows from (5), (6), (9) and (10) that

$$
\begin{gathered}
\left(1+3 \lambda-2 \lambda^{2}\right) a_{2}=B_{1} b_{1}, \\
\left(12 \lambda^{4}-28 \lambda^{3}+11 \lambda^{2}+2 \lambda-1\right) a_{2}^{2}+\left(4 \lambda^{2}+2\right) a_{3}=B_{1} b_{2}+B_{2} b_{1}^{2},
\end{gathered}
$$

and

$$
\begin{gathered}
-\left(1+3 \lambda-2 \lambda^{2}\right) a_{2}=B_{1} c_{1}, \\
\left(12 \lambda^{4}-28 \lambda^{3}+19 \lambda^{2}+2 \lambda+3\right) a_{2}^{2}-\left(4 \lambda^{2}+2\right) a_{3}=B_{1} c_{2}+B_{2} c_{1}^{2} .
\end{gathered}
$$

From (11) and (13) we obtain

$$
c_{1}=-b_{1} .
$$

By adding (14) to (12), further computations using (11) to (15) lead to

$$
\left[2\left(12 \lambda^{4}-28 \lambda^{3}+15 \lambda^{2}+2 \lambda+1\right) B_{1}^{2}-2\left(1+3 \lambda-2 \lambda^{2}\right)^{2} B_{2}\right] a_{2}^{2}=B_{1}^{3}\left(b_{2}+c_{2}\right) \text {. }
$$

(15) and (16), together with (4), give that

$$
\left|\left(12 \lambda^{4}-28 \lambda^{3}+15 \lambda^{2}+2 \lambda+1\right) B_{1}^{2}-\left(1+3 \lambda-2 \lambda^{2}\right)^{2} B_{2}\right|\left|a_{2}\right|^{2} \leq B_{1}^{3}\left(1-\left|b_{1}\right|^{2}\right) .
$$

From (11) and (17) we get

$$
\left|a_{2}\right| \leq \frac{B_{1} \sqrt{B_{1}}}{\sqrt{\left|\left(12 \lambda^{4}-28 \lambda^{3}+15 \lambda^{2}+2 \lambda+1\right) B_{1}^{2}-\left(1+3 \lambda-2 \lambda^{2}\right)^{2} B_{2}\right|+\left(1+3 \lambda-2 \lambda^{2}\right)^{2} B_{1}}} .
$$

Next, in order to find the bound on $\left|a_{3}\right|$, by subtracting (14) from (12), we obtain

$$
4\left(2 \lambda^{2}+1\right) a_{3}-4\left(2 \lambda^{2}+1\right) a_{2}^{2}=B_{1}\left(b_{2}-c_{2}\right)+B_{2}\left(b_{1}^{2}-c_{1}^{2}\right) .
$$


Then, in view of (4) and (15), we have

$$
2\left(2 \lambda^{2}+1\right) B_{1}\left|a_{3}\right| \leq\left[2\left(2 \lambda^{2}+1\right) B_{1}-\left(1+3 \lambda-2 \lambda^{2}\right)^{2}\right]\left|a_{2}\right|^{2}+B_{1}^{2} .
$$

Notice that (7), we get

$$
\left|\mathrm{a}_{3}\right| \leq\left\{\begin{array}{c}
\frac{\mathrm{B}_{1}}{2\left(2 \lambda^{2}+1\right)} ; \quad \text { if } \mathrm{B}_{1} \leq \frac{\left(1+3 \lambda-2 \lambda^{2}\right)^{2}}{2\left(2 \lambda^{2}+1\right)} \\
\frac{\left|\left(12 \lambda^{4}-28 \lambda^{3}+15 \lambda^{2}+2 \lambda+1\right) \mathrm{B}_{1}^{2}-\left(1+3 \lambda-2 \lambda^{2}\right)^{2} \mathrm{~B}_{2}\right| \mathrm{B}_{1}+2\left(2 \lambda^{2}+1\right) \mathrm{B}_{1}^{3}}{2\left(2 \lambda^{2}+1\right)\left[\left|\left(12 \lambda^{4}-28 \lambda^{3}+15 \lambda^{2}+2 \lambda+1\right) \mathrm{B}_{1}^{2}-\left(1+3 \lambda-2 \lambda^{2}\right)^{2} \mathrm{~B}_{2}\right|+\left(1+3 \lambda-2 \lambda^{2}\right)^{2} \mathrm{~B}_{1}\right]} \\
\text { if } \mathrm{B}_{1}>\frac{\left(1+3 \lambda-2 \lambda^{2}\right)^{2}}{2\left(2 \lambda^{2}+1\right)} .
\end{array}\right.
$$

Putting $\lambda=0$ in Theorem 1, we have the following corollary.

Corollary 1 Let $\mathrm{f}$ given by (1) be in the class $\mathrm{S}_{\Sigma}^{*}(\phi)$. Then

$$
\left|a_{2}\right| \leq \frac{B_{1} \sqrt{B_{1}}}{\sqrt{\left|B_{1}^{2}-B_{2}\right|+B_{1}}}
$$

and

$$
\left|a_{3}\right| \leq\left\{\begin{array}{ccc}
\frac{B_{1}}{2} ; & \text { if } & B_{1} \leq \frac{1}{2} \\
\frac{\left|B_{1}^{2}-B_{2}\right| B_{1}+2 B_{1}^{3}}{2\left[\left|B_{1}^{2}-B_{2}\right|+B_{1}\right]} ; & \text { if } & B_{1}>\frac{1}{2} .
\end{array}\right.
$$

The estimates on the coefficients $\left|a_{2}\right|$ and $\left|a_{3}\right|$ of Corollary 1 are improvement of the estimates obtained in Corollary 2.1 in [19].

Corollary 2 If let

$$
\phi(z)=\left(\frac{1+z}{1-z}\right)^{\alpha}=1+2 \alpha z+2 \alpha^{2} z^{2}+\ldots \quad(0<\alpha \leq 1),
$$

then inequalities (7) and (8) become

$$
\left|a_{2}\right| \leq \frac{2 \alpha}{\sqrt{\left|20 \lambda^{4}-44 \lambda^{3}+25 \lambda^{2}-2 \lambda+1\right| \alpha+\left(1+3 \lambda-2 \lambda^{2}\right)^{2}}}
$$


and

$$
\left|a_{3}\right| \leq\left\{\begin{array}{cc}
\frac{\alpha}{2 \lambda^{2}+1} ; & \text { if } 0<\alpha \leq \frac{\left(1+3 \lambda-2 \lambda^{2}\right)^{2}}{4\left(2 \lambda^{2}+1\right)} \\
\frac{\left[\left|20 \lambda^{4}-44 \lambda^{3}+25 \lambda^{2}-2 \lambda+1\right|+4\left(2 \lambda^{2}+1\right)\right] \alpha^{2}}{\left(2 \lambda^{2}+1\right)\left[20 \lambda^{4}-44 \lambda^{3}+25 \lambda^{2}-2 \lambda+1 \mid \alpha+\left(1+3 \lambda-2 \lambda^{2}\right)^{2}\right]} ; & \text { if } \frac{\left(1+3 \lambda-2 \lambda^{2}\right)^{2}}{4\left(2 \lambda^{2}+1\right)}<\alpha \leq 1 .
\end{array}\right.
$$

The bounds on $\left|\mathrm{a}_{2}\right|$ and $\left|\mathrm{a}_{3}\right|$ given by (19) and (20) are more accurate than that given in Theorem 2.1 in [15].

We note that for $\lambda=0$, the class $S_{\Sigma}(\lambda, \phi)$ reduces to the class of strongly bi-starlike functions of order $\alpha(0<\alpha \leq 1)$ and denoted by $S_{\Sigma}^{\star}(\alpha)$.

Putting $\lambda=0$ in Corollary 2, we have the following corollary.

Corollary 3 Let $f$ given by (1) be in the class $S_{\Sigma}^{*}(\alpha),(0<\alpha \leq 1)$. Then

$$
\left|a_{2}\right| \leq \frac{2 \alpha}{\sqrt{\alpha+1}}
$$

and

$$
\left|\mathrm{a}_{3}\right| \leq\left\{\begin{array}{c}
\alpha ; \quad \text { if } 0<\alpha \leq \frac{1}{4} \\
\frac{5 \alpha^{2}}{\alpha+1} ; \quad \text { if } \frac{1}{4}<\alpha \leq 1 .
\end{array}\right.
$$

The bounds on $\left|\mathrm{a}_{3}\right|$ given by (22) is more accurate than that given by Remark 2.2 in [17] and Theorem 2.1 in [4].

Remark 1 The bounds on $\left|\mathrm{a}_{3}\right|$ given by (22) is more accurate than that given in Corollary 2.3 in [18].

Corollary 4 If let

$$
\phi(z)=\frac{1+(1-2 \alpha) z}{1-z}=1+2(1-\alpha) z+2(1-\alpha) z^{2}+\cdots \quad(0<\alpha \leq 1),
$$

then inequalities (7) and (8) become

$$
\left|a_{2}\right| \leq \frac{2(1-\alpha)}{\sqrt{\left|2(1-\alpha)\left(12 \lambda^{4}-28 \lambda^{3}+15 \lambda^{2}+2 \lambda+1\right)-\left(1+3 \lambda-2 \lambda^{2}\right)^{2}\right|+\left(1+3 \lambda-2 \lambda^{2}\right)^{2}}}
$$


and

$$
\left|a_{3}\right| \leq \begin{cases}\frac{1-\alpha}{2 \lambda^{2}+1} ; & \text { if } \frac{4\left(2 \lambda^{2}+1\right)-\left(1+3 \lambda-2 \lambda^{2}\right)^{2}}{4\left(2 \lambda^{2}+1\right)} \leq \alpha<1 \\ \frac{\left[\left|2(1-\alpha)\left(12 \lambda^{4}-28 \lambda^{3}+15 \lambda^{2}+2 \lambda+1\right)-\left(1+3 \lambda-2 \lambda^{2}\right)^{2}\right|+4(1-\alpha)\left(2 \lambda^{2}+1\right)\right](1-\alpha)}{\left(2 \lambda^{2}+1\right)\left[\left|2(1-\alpha)\left(12 \lambda^{4}-28 \lambda^{3}+15 \lambda^{2}+2 \lambda+1\right)-\left(1+3 \lambda-2 \lambda^{2}\right)^{2}\right|+\left(1+3 \lambda-2 \lambda^{2}\right)^{2}\right]} & \text { if } 0 \leq \alpha<\frac{4\left(2 \lambda^{2}+1\right)-\left(1+3 \lambda-2 \lambda^{2}\right)^{2}}{4\left(2 \lambda^{2}+1\right)}\end{cases}
$$

The bounds on $\left|\mathrm{a}_{2}\right|$ and $\left|\mathrm{a}_{3}\right|$ given by (23) and (24) are more accurate than that given in Theorem 3.1 in [15].

Putting $\lambda=0$ in Corollary 4, we have the following corollary.

Corollary 5 Let $f$ given by (1) be in the class $S_{\Sigma}^{\star}(\alpha),(0 \leq \alpha<1)$. Then

$$
\left|a_{2}\right| \leq \frac{2(1-\alpha)}{\sqrt{1+|1-2 \alpha|}}
$$

and

$$
\left|\mathrm{a}_{3}\right| \leq\left\{\begin{array}{cl}
1-\alpha ; & \text { if } \frac{3}{4} \leq \alpha<1 \\
\frac{(1-\alpha)|1-2 \alpha|+4(1-\alpha)^{2}}{1+|1-2 \alpha|} ; & \text { if } 0 \leq \alpha<\frac{3}{4} .
\end{array}\right.
$$

The bounds on $\left|\mathrm{a}_{3}\right|$ given by (26) is more accurate than that given by Remark 2.2 in [17] and Theorem 3.1 in [4].

Remark 2 The bounds on $\left|\mathrm{a}_{3}\right|$ given by (26) is more accurate than that given in Corollary 3.3 in [18].

\section{Acknowledgements}

The authors are extremely grateful to the reviewers for a careful reading of the manuscript and making valuable suggestions leading to a better presentation of the paper. 


\section{References}

[1] S. Altinkaya, S. Yalcin, Initial coefficient bounds for a general class of biunivalent functions, International Journal of Analysis, Article ID 867871, (2014), 4 pp.

[2] S. Altinkaya, S. Yalcin, Coefficient bounds for a subclass of bi-univalent functions, TWMS Journal of Pure and Applied Mathematics, in press.

[3] S. Altinkaya, S. Yalcin, Fekete-Szegö Inequalities for certain classes of biunivalent functions, International Scholarly Research Notices, Article ID 327962, (2014) 6 pp.

[4] D. A. Brannan, T. S. Taha, On some classes of bi-univalent functions, Studia Universitatis Babeş-Bolyai. Mathematica, 31 (2) (1986), 70-77.

[5] D. A. Brannan, J. G. Clunie, Aspects of comtemporary complex analysis, (Proceedings of the NATO Advanced Study Institute, Held at University of Durham: July 1-20, 1979). New York: Academic Press, (1980).

[6] S. Bulut, Faber polynomial coefficient estimates for a comprehensive subclass of analytic bi-univalent functions, C. R. Acad. Sci. Paris, Ser. I, in press.

[7] O. Crişan, Coefficient estimates for certain subclasses of bi-univalent functions, Gen. Math. Notes, 16 (2) (2013), 93-102.

[8] P. L. Duren, Univalent Functions, Grundlehren der Mathematischen Wissenschaften, Springer, New York, NY, USA, 259 (1983).

[9] B. A. Frasin, M. K. Aouf, New subclasses of bi-univalent functions, Applied Mathematics Letters, 24 (2011), 1569-1573.

[10] S. G. Hamidi and J. M. Jahangiri, Faber polynomial coefficient estimates for analytic bi-close-to-convex functions, C. R. Acad. Sci. Paris, Ser.I V. 352 (1) (2014), pp. 17-20.

[11] J. M. Jahangiri, S. G. Hamidi, Coefficient estimates for certain classes of bi-univalent functions, Int. J. Math. Math. Sci., ArticleID 190560, (2013), 4 pp.

[12] J. M. Jahangiri, S. G. Hamidi, S. A. Halim, Coefficients of bi-univalent functions with positive real part derivatives, Bull. Malays. Math. 
Soc., in press, http://math.usm.my/bulletin/pdf/acceptedpapers/201304-050-R1.pdf.

[13] B. Srutha Keerthi, Bhuvaneswari Raja, Coefficient inequality for certain new subclasses of analytic bi-univalent functions, Theoretical Mathematics and Applications, 3 (1) (2013), pp. 1-10.

[14] M. Lewin, On a coefficient problem for bi-univalent functions, Proceeding of the American Mathematical Society, 18 (1967), 63-68.

[15] N. Magesh and J. Yamini, Coefficient bounds for a certain subclass of bi-univalent functions, International Mathematical Forum, 8 (27) (2013), $1337-1344$.

[16] E. Netanyahu, The minimal distance of the image boundary from the orijin and the second coefficient of a univalent function in $|z|<1$, Archive for Rational Mechanics and Analysis, 32 (1969), 100-112.

[17] Z. Peng and Q. Han, On the coefficients of several classes of bi-univalent functions, Acta Mathematica Scientia, 34B (1) (2014), 228-240.

[18] S. Prema, B. Srutha Keerthi, Coefficient bounds for a certain subclass of analytic functions, Journal of Mathematical Analysis, 4 (1) (2013), 22-27.

[19] R. M. Ali, S. K. Lee, V. Ravichandran, S. Supramaniam, Coefficient estimates for bi-univalent Ma-Minda starlike and convex functions, Applied Mathematics Letters, 5 (2012), 344-351.

[20] H. M. Srivastava, A. K. Mishra, P. Gochhayat, Certain subclasses of analytic and bi-univalent functions, Applied Mathematics Letters, 23 (10), $1188-1192$.

[21] H. M. Srivastava, S. Bulut, M. Çağlar, N. Yağmur, Coefficient estimates for a general subclass of analytic and bi-univalent functions, Filomat, 27 (5)(2013), 831-842.

[22] Q. H. Xu, Y. C. Gui, H. M. Srivastava, Coefficient estimates for a certain subclass of analytic and bi-univalent functions, Applied Mathematics Letters, 25 (2012), 990-994. 